\title{
Le classement des digues littorales au titre de la sécurité civile : un exemple de mise en oeuvre en Vendée
}

\author{
Stéphane RAISON ${ }^{1}$ \\ ${ }^{1}$ Direction Départementale de 1'Equipement de la Vendée, Service Maritime et \\ des Risques , 1 quai Dingler - BP 10366, 85108 Les Sables d'Olonne Cedex \\ stephane.raison@developpement-durable.gouv.fr
}

\section{Résumé :}

Le littoral vendéen poldérisé depuis le $17^{\text {ème }}$ siècle a un trait de côte figé sur plus de $100 \mathrm{~km}$ de linéaire. La protection de zones vulnérables est principalement assurée par un réseau de digues en front de mer. Les conséquences des grandes tempêtes de 1940, et plus récemment celle de fin 1999, ont rendu nécessaire un renforcement des politiques publiques pour les ouvrages de protection le long du littoral. Cette communication illustre la mise en œuvre du classement au titre de la sécurité civile d'une digue en terre de $6 \mathrm{~km}$, située dans le sud du département et qui assure la protection d'une commune dont la totalité du territoire bâti se situe en deçà des niveaux extrêmes de la mer. Différents aspects seront abordés : la définition des aléas, les enjeux, l'évaluation de l'état structurel de l'ouvrage et la gestion administrative de la démarche.

\begin{abstract}
:
Vendée's shoreline, backed by polders from the $17^{\text {th }}$ century, is artificially fixed along more than $100 \mathrm{~km}$. Areas at risk are mainly protected by a network of seawalls. The consequences of the major storms in 1940 and more recently at the end of 1999 made it necessary to reinforce public action for coastal protection structures. This article highlights the categorization of a $6 \mathrm{~km}$-long embankment. Located in southern Vendée, it protects a town that is totally built under the highest sea levels. The definition of hazards and stakes, the structural aspect evaluation and the administrative side of the categorization are developed.
\end{abstract}

Mots-clés :

Génie côtier - Environnement littoral - Risques littoraux

\section{Introduction}

Le long des $276 \mathrm{~km}$ de côtes du département de la Vendée, de vastes espaces poldérisés sont protégés par $103 \mathrm{~km}$ de digues à la mer. Plusieurs inondations 
importantes sont intervenues dans tous les secteurs du littoral. Les plus récentes et tristement célèbres restent la rupture du Polder de Sébastopol sur l'île de Noirmoutier en 1979 avec plus de 3000 hectares submergés et l'ouragan de janvier 1940 avec la destruction des digues du polder de Bouin. Plus récemment, les tempêtes du mois de décembre 1999 ont démontré que les zones côtières pouvaient être submergées par la mer notamment en zone estuarienne. La mer a ainsi atteint un niveau exceptionnellement élevé par la combinaison d'une marée de coefficient moyen (78), d'un vent violent et d'une forte dépression barométrique. Les surcotes ayant été très importantes (plus de $2 \mathrm{~m}$ mesurés à La Rochelle), certaines digues n'étaient pas assez hautes et ont été submergées ou se sont rompues.

Dans le cadre de la mise en place des Plans de Prévention des Risques Littoraux, la Direction Départementale de l'Equipement de la Vendée a, dès l'année 2005, souhaité accompagner son action réglementaire, d'une part par des actions de connaissance et d'autre part par le renforcement de prescriptions sur les digues à la mer. Une démarche pilote a été engagée avec les services préfectoraux pour encadrer réglementairement les ouvrages de défense contre la mer et favoriser leur contrôle avec éventuellement des travaux de réparation ou de mise en conformité.

L'article fait un état des lieux précis de l'historique de la démarche, le diagnostic de la situation, les enjeux et le plan de travail des services.

\section{Histoire des évènements majeurs de submersion marine en Vendée}

\subsection{La mémoire du risque : un élément à raviver dans les consciences collectives}

De tous temps, les submersions marines ont marqué le territoire littoral. Sur l'île de Noirmoutier par exemple, la mémoire du risque a été entretenue par les associations locales qui ont largement travaillé sur ce thème (voir tableau 1). Depuis l'an mil, l'île a été périodiquement assaillie par des tempêtes ou des ouragans dont les recueils des paroisses ont gardé une trace. Plus récemment les événements de 1979 ou 1999, ont menacé clairement les zones habitées.

Sur le secteur sud du département, les submersions recouvrent lors des grands évènements le Nord de la pointe d'Arçay. Les secteurs de la Vieille Prise et la Jeune Prise, à partir de l'Ouest à travers le secteur du Platin étaient submergés le 21 mars 1928. La surface inondée d'environ 120 hectares coupait la route de La Faute-sur-Mer, et le village de la Faute est devenu une île. Une submersion similaire, partant de l'Ouest et rejoignant le Lay, avait également eu lieu plus au Nord en 1906. Vers 1930, de fortes attaques se sont produites un peu plus au Sud, 
démolissant une digue d'une longueur de $800 \mathrm{~m}$. En 1940, la rupture de la digue des Wagons à Triaize a submergé environ 3000 hectares de terres aménagées et cultivées. En l'espèce, sur ce même estuaire du Lay, une digue de protection a cédé à l'automne 1999 sur le territoire de la commune voisine de l'Aiguillon-surMer, juste de l'autre côté de l'estuaire sur la rive gauche du Lay. Cette rupture a entraîné l'inondation d'une trentaine d'hectares de terres agricoles.

Tableau 1. Les submersions marines sur l'île de Noirmoutier (Association 12/12)

\begin{tabular}{|c|c|}
\hline $\begin{array}{l}\text { Les i } \\
\text { surv }\end{array}$ & $\begin{array}{l}\text { ations : quelques évènements extraits du répertoire des catastrop } \\
\text { dans l'Ile de Noirmoutier }\end{array}$ \\
\hline 1075 & Un fort « vimer » envahit les champs au Both et au Fier. \\
\hline 1351 & $\begin{array}{l}\text { Reprise par la mer de très grands territoires dans toute la plaine de } \\
\text { Barbâtre. }\end{array}$ \\
\hline 1509 & $\begin{array}{l}\text { Ouragan : la mer rompt la digue de Pulant et envahit la plaine de la } \\
\text { Guérinière. }\end{array}$ \\
\hline 1638 & Raz-de-marée : une partie de l'île est inondée. \\
\hline 1762 & Nouveau désastre : l'île est menacée d'une inondation générale. \\
\hline 1763 & $\begin{array}{l}\text { Le } 3 \text { février, brèche aux dunes du Devin. Les digues de la Frandière sont } \\
\text { rompues en trois endroits; la mer noie toutes les terres de la Fosse, de la } \\
\text { Frandière et des Onchères; le moulin des Onchères et un certain nombre } \\
\text { de maisons, dont une dizaine du bourg, sont engloutis et dévastés. (Pages } \\
\text { d'Histoire noirmoutrine - Fernand Guillet, 1948). }\end{array}$ \\
\hline 1838 & $\begin{array}{l}\text { Destruction des digues privées sur la côte de Pulant, inondations } \\
\text { catastrophiques à la Guérinière. Il faut des barques pour passer d'une } \\
\text { maison à l'autre. }\end{array}$ \\
\hline 1882 & $\begin{array}{l}\text { Les travaux d'endiguement au niveau du village de la Guérinière sont } \\
\text { insuffisants contre les tempêtes du Sud-Ouest. Déjà la mer y est arrivée } \\
\text { avec un courant de foudre et presque toujours en pleine nuit, envahissant } \\
\text { l'église, la cure et les maisons voisines. }\end{array}$ \\
\hline 1926 & $\begin{array}{l}\text { Le } 20 \text { novembre, « lors d'une effroyable tempête, la mer coupe les dunes } \\
\text { de Bressuire (l'Epine) et rentre à flots jusqu'aux abords du village ». }\end{array}$ \\
\hline 1937 & $\begin{array}{l}\text { Rupture de la digue à la Tresson : la mer atteint la route }(\mathrm{N} \text { 148), } 130 \text { ha } \\
\text { inondés. Dans la nuit du } 13 \text { au } 14 \text { mars, les habitants de l'île de } \\
\text { Noirmoutier et des communes du marais de Monts et de Bouin furent } \\
\text { alertés par le tocsin. Coefficient de marée de } 85 \text {, le baromètre descend }\end{array}$ \\
\hline
\end{tabular}




\begin{tabular}{|l|l|}
\hline 1996 & $\begin{array}{l}\text { brusquement, surcote d'au moins } 1 \mathrm{~m} . \\
\text { Vents violents et mer très forte. Durant les mois de janvier et de février, (en } \\
\text { particulier le } 7 \text { février), toutes les côtes de l'île subissent des dégâts } \\
\text { importants. }\end{array}$ \\
\hline 1999 & $\begin{array}{l}\text { Le } 24 \text { octobre, la conjonction d'une forte dépression, d'un coefficient de } \\
\text { marée élevé (coefficient de 102, hauteur d'eau de } 6,15 \text { m à Saint-Nazaire } \\
\text { soit environ 5,75 dans le port de Noirmoutier) et de vents violents de } \\
\text { secteur Ouest-Sud-Ouest provoque une surcote exceptionnelle d'environ } \\
\text { 0,9 m. Les digues sont touchées et des débordements ont lieu au niveau des } \\
\text { berges d'étiers et sur les quais du port de Noirmoutier. }\end{array}$ \\
\hline 1999 & $\begin{array}{l}\text { Les 26 et 27 décembre, même scénario : le coefficient supérieur à } 100 \text { qui } \\
\text { accompagne la tempête amplifie l'action érosive de la mer. }\end{array}$ \\
\hline
\end{tabular}
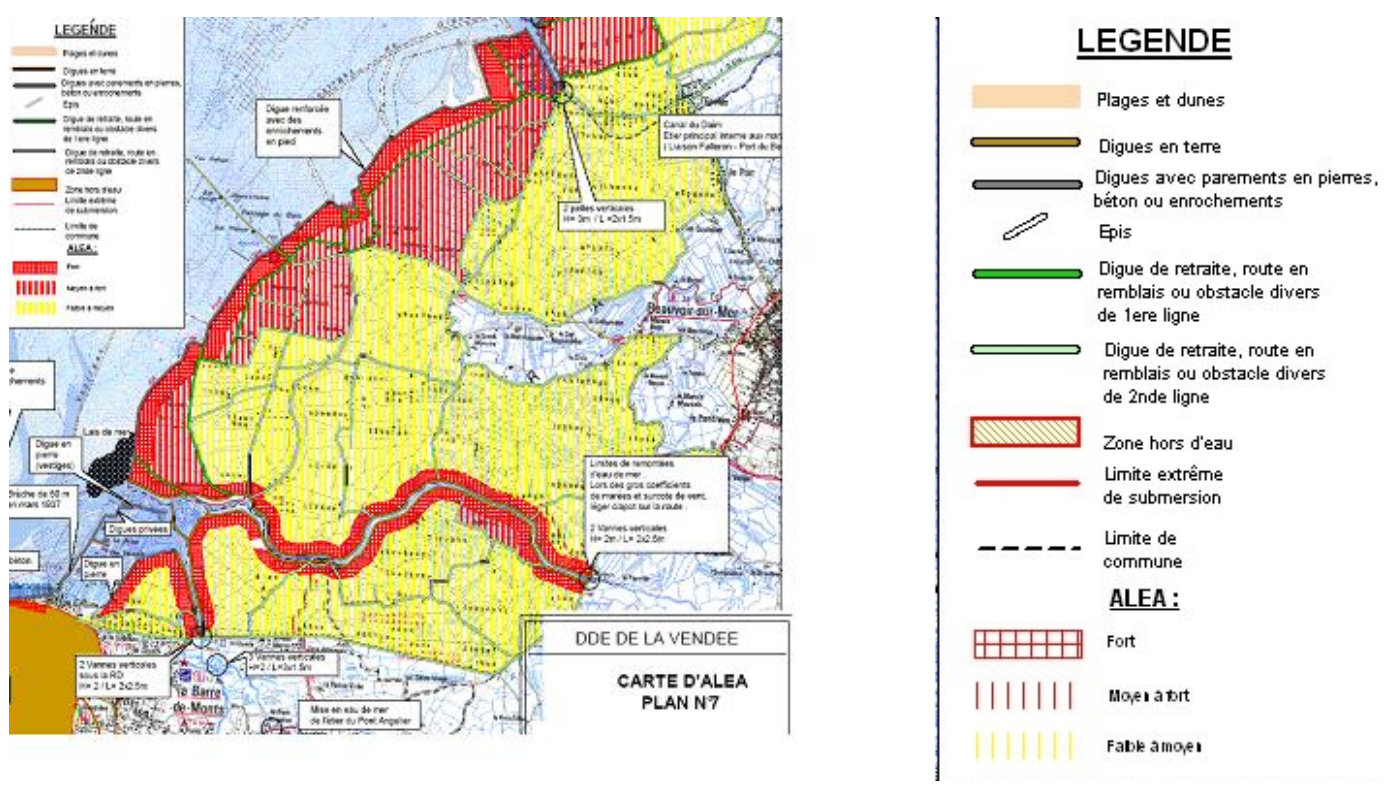

Figure 1. L'atlas de submersion marine des digues de la baie de Bourgneuf (DDE de la Vendée et SOGREAH, 2002)

\subsection{L'atlas de l'aléa submersion marine sur le littoral vendéen : un premier élément de connaissance}

La vulnérabilité du littoral vendéen aux submersions marines ne fait aucun doute. A partir de l'année 2000, les services de l'Etat travaillent à l'élaboration d'un atlas des zones de submersion marine. Sur la base d'un aléa de référence fixé à $4 \mathrm{~m} \mathrm{NGF}$, les zones submersibles sont calculées et cartographiées.

Cette cartographie est diffusée à l'ensemble des communes, elle sert de base à la 
mise en place d'un Plan de Prévention des Risques de submersion marine dans l'estuaire du Lay sur les communes de La Faute-sur-Mer et de l'Aiguillon-surMer, où la conjonction de deux phénomènes, de crue dans l'estuaire du Lay et de submersion marine, pourrait avoir un impact très important sur les zones densifiées à l'arrière d'un réseau de digues vieillissant.

Sur le secteur littoral, la zone de l'estuaire du Lay est la zone la plus dangereuse $\mathrm{du}$ département, le Lay étant un cours d'eau majeur drainant une surface représentant la moitié du département de la Vendée, exposée à des phénomènes marins extrêmes amplifiés par l'effet de baie dans la baie de l'Aiguillon.

\subsection{L'élément déclencheur : le cas de la Faute sur Mer}

La commune de La Faute-sur-Mer dans le sud-Vendée est une commune jeune. Rattachée à la commune de la Tranche-sur-Mer jusqu'en novembre 1945, elle a été construite sur de vastes espaces gagnés sur la mer, ne tenant pas compte de la mémoire du risque (voir figure 2). En effet, après les grandes tempêtes de 1926 et 1929, un réseau de digues de protection est créé le long de l'estuaire du Lay. D'une longueur de près de $5750 \mathrm{~m}$, la digue dite «Est » a été réalisée en terre en utilisant les matériaux du site. Elle a été entretenue dans le temps par apport de matériaux divers sans contrôle de leur qualité ni de leur provenance. Cet ouvrage n'a jamais fait l'objet d'un diagnostic approfondi de la part du maître d'ouvrage.

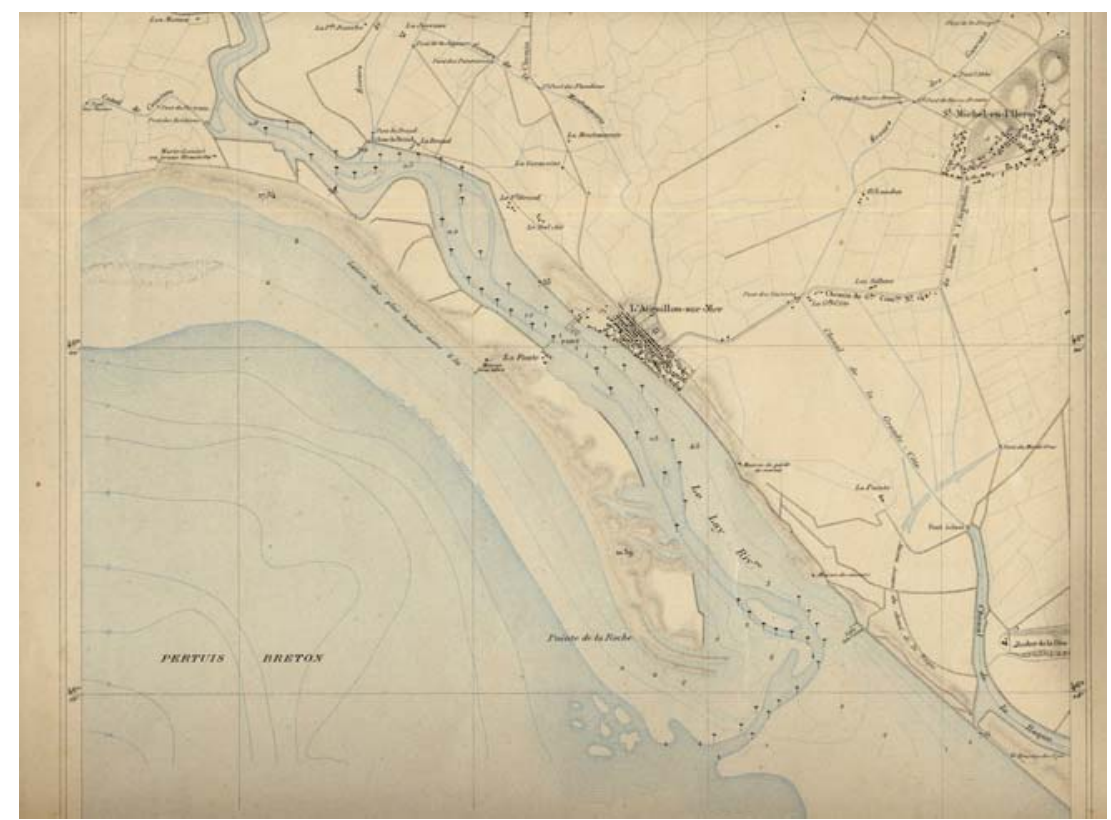

Figure 2. Les communes de La Faute-sur-Mer et l'Aiguillon en 1878 (DDE de la Vendée)

La commune s'est urbanisée de façon importante dans les années 1980 et aujourd'hui plus de 3000 maisons sont construites derrière ces digues en terre. 
40000 personnes fréquentent quotidiennement la commune en été. La rupture des digues sur ce secteur engendrerait des dégâts majeurs aux biens et aux personnes en regard de la carte des aléas, présentée en figure 3. La D.D.E. exerce, au titre de ses missions de police des eaux et en application d'une circulaire du Ministère de l'Ecologie du 6 août 2003, le contrôle des digues intéressant la sécurité publique pour les digues fluviales et les digues bordant le Domaine Public Fluvial géré par la DDE (cours aval du Lay et de la Vie, canal de Luçon).

Sur la base de cet inventaire et par l'examen des enjeux (personnes, biens, routes à grande circulation) en arrière des digues, la DDE a répertorié en 2005 les digues pouvant être classées comme intéressant la sécurité civile au sens de la circulaire sus-visée en augmentant son champ d'action aux digues à la mer.

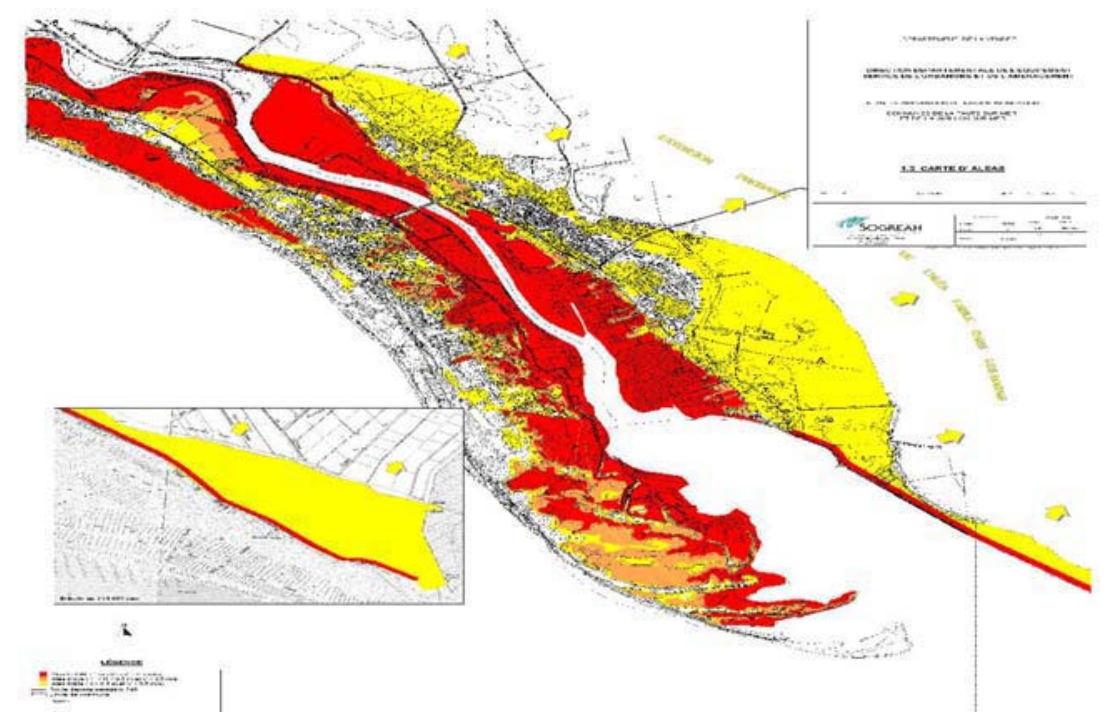

Figure 3. Aléa de submersion et d'inondation marine dans l'estuaire du Lay. PPR du Lay aval (DDE de la Vendée et SOGREAH, 2003)

Sur la base de la connaissance de l'aléa, la DDE a donc établi la définition des enjeux sur tous les secteurs protégés par des digues. Lorsque les enjeux humains liés à la rupture des digues étaient jugés caractérisés comme importants (zone rouge et population impliquée importante ou hauteur d'eau supérieure à $1 \mathrm{~m}$ ), les digues ont été proposées au classement (voir figure 4).

Sur cette base, les cartes d'exposition des personnes aux submersions ont été établies et $42 \mathrm{~km}$ de digues à la mer ont été proposées au classement (voir figure 5). Compte tenu des éléments de connaissance obtenus par l'Etat, la digue Est de la Faute-sur-Mer (voir figure 6) a été la première du département à être classée comme ayant un intérêt pour la sécurité publique et à faire l'objet de prescriptions de diagnostic, de surveillance et d'entretien, par arrêté préfectoral du 7 juillet 2005 . 


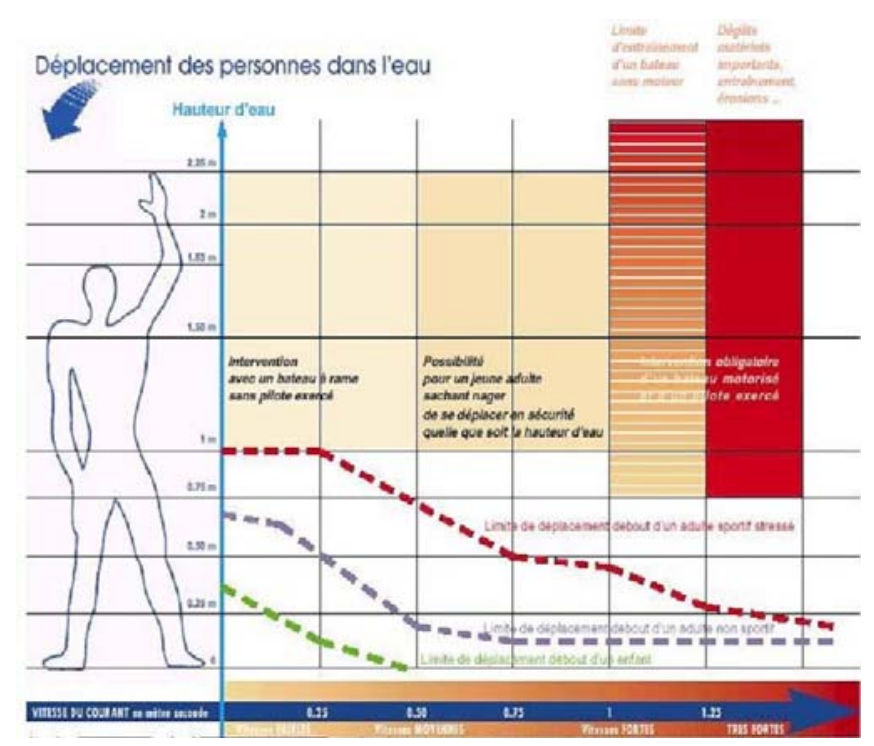

Figure 4. Graphique des dangers de noyade (MEDD/DPPR/SDPRM)

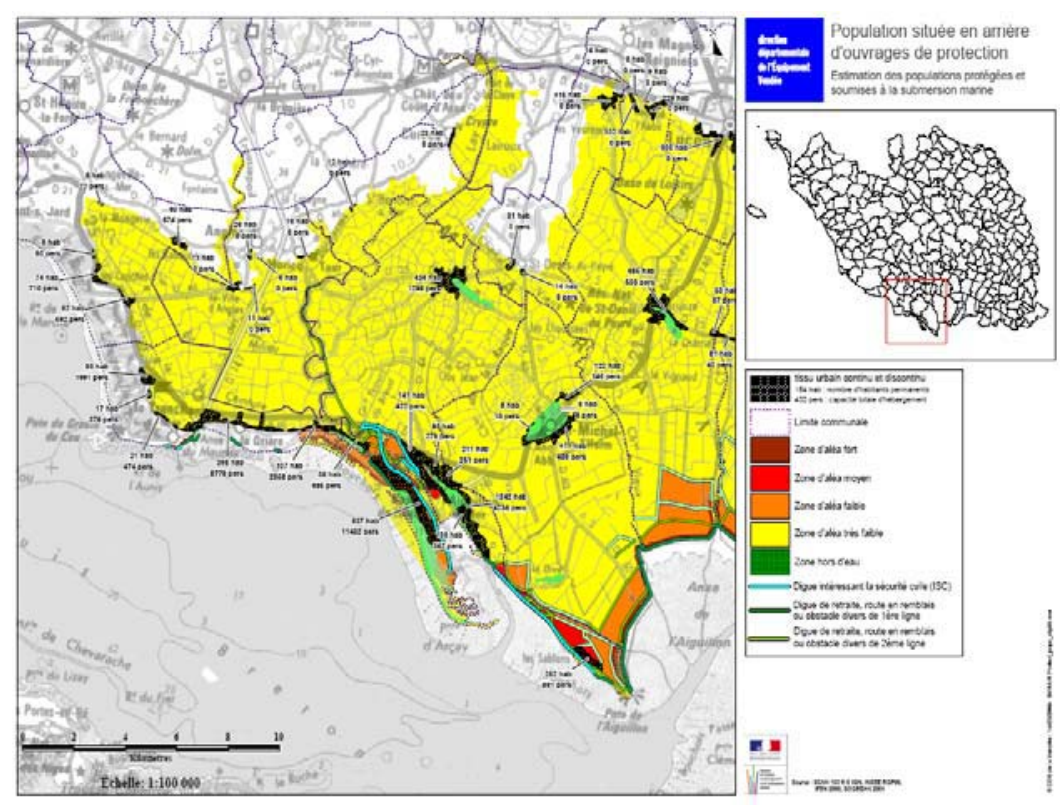

Figure 5. Définition des populations exposées (DDE de la Vendée / SMR, 2006)

Nonobstant les éléments sur la dangerosité du site, cette commune était dans une situation particulière pour la maîtrise d'ouvrage des travaux. Le propriétaire actuel (association syndicale des marais de La Faute-sur-Mer), le propriétaire futur qu'est la commune, qui finance déjà tous les travaux, et une association syndicale de la vallée du Lay qui exécute les travaux ne permettaient pas de réaliser une politique coordonnée de protection et de suivi des ouvrages. Une action complémentaire a donc été menée sur la reconfiguration des maîtrises d'ouvrages. 


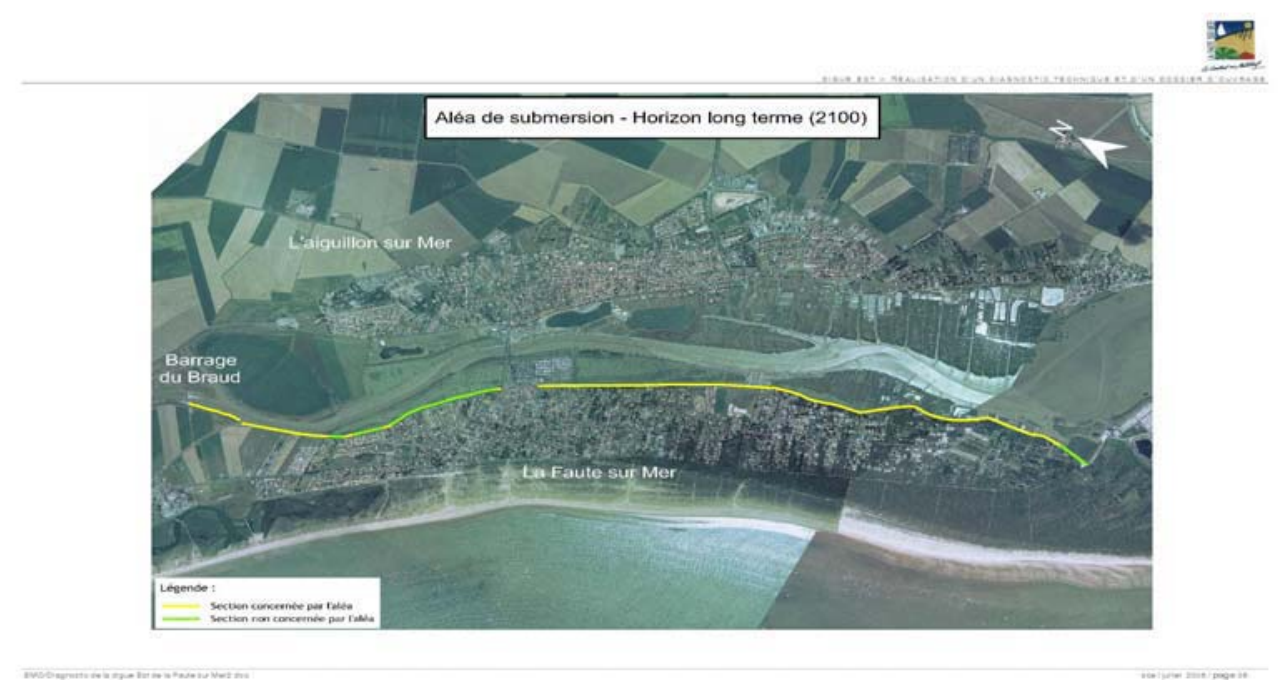

Figure 6. Digue Est de la Faute sur Mer (DDE et SCE, 2005)

\subsection{Le contenu des arrêtés de classement au titre de la sécurité civile}

Les arrêtés ont été structurés de la sorte : le propriétaire de la digue constitue, dans un délai de trois mois après la date de signature du présent arrêté, le dossier de l'ouvrage contenant les documents administratifs et techniques sur les ouvrages ainsi que les documents de gestion. Le propriétaire de la digue est tenu de mettre en place un dispositif de surveillance adapté à la nature de l'ouvrage, à ses dimensions et à son intérêt pour la sécurité publique. A ce titre, le propriétaire :

- établit des consignes permanentes de surveillance et d'entretien de l'ouvrage et de ses annexes, y compris des organes de vidanges (s'il en existe), portant notamment sur le contrôle de la végétation, l'entretien des accès et les mesures à prendre en cas de désordres et lors des périodes à risques. Il s'appuie pour cela sur les dispositions de l'étude technique ;

- effectue des visites périodiques portant sur l'examen visuel de l'ouvrage, de ses abords ;

- signale sans délai au maire et au service de police de l'eau toute anomalie constatée lors des visites.

Dans le cadre de ce dispositif de surveillance, le propriétaire s'organise avec les tiers propriétaires de réseaux ou d'ouvrages traversant sa digue afin que ceux-ci assurent une surveillance et un entretien réguliers de leurs propres ouvrages permettant de ne pas affaiblir la sécurité générale de la digue. Dans tous les cas, le propriétaire de la digue demeure seul responsable de la sécurité générale de la digue.

A compter de la date de notification du présent arrêté le propriétaire de la digue tient, dans des locaux occupés hors de portée de toute inondation, un registre sur lequel figureront les consignes permanentes de surveillance et d'entretien établies 
et seront mentionnés au fur et à mesure, avec indication des dates, les principaux renseignements relatifs aux visites d'inspection, aux incidents constatés (fuites, fissures, renards...) et les travaux d'entretien et de réparation effectués.

Tous les ans, le propriétaire de la digue envoie au service de police de l'eau et au maire un rapport sur la surveillance et l'entretien de l'ouvrage. Le propriétaire fournit au service de police de l'eau dans un délai maximal d'un an à compter de la date de signature du présent arrêté :

- une étude permettant de déterminer la durée de retour des risques de surverse et analysant le fonctionnement de l'ouvrage selon divers scénarios correspondant à ces évènements hydrauliques de référence et à des évènements supérieurs et/ou correspondant à des ruptures de digues; cette étude redéfinit le dispositif de surveillance ;

- une étude appuyée notamment sur un diagnostic approfondi permettant d'apprécier les faiblesses de l'ouvrage et de définir les travaux nécessaires à sa remise en état et à son entretien.

A partir de l'étude initiale, si l'ouvrage apparaît en bon état, ou de la visite après travaux dans le cas contraire, des visites trimestrielles sont effectuées par le propriétaire. Elles comportent notamment un examen visuel de la digue et le contrôle de l'état de fonctionnement des ouvrages. Le compte-rendu de ces visites périodiques est intégré au rapport annuel.

Une visite de la digue est effectuée par le propriétaire après chaque événement l'ayant sollicitée de manière significative. Elle s'appuie sur les éléments de contrôle technique définis par les études techniques. Un compte-rendu de cette visite est intégré au rapport annuel.

Une visite décennale, à partir de la visite initiale ou d'une toute autre visite complète, est effectuée par le service de police de l'eau en présence du propriétaire dûment convoqué. L'objectif d'une telle visite est d'inspecter toutes les parties de la digue et de ses organes annexes. Les points à observer et les relevés de désordres éventuels sont précisés notamment dans l'étude technique initiale.

Dans le cas de la Faute-sur-Mer, les études ont été menées dans le délais prescrits par les services de l'Etat. Les études ont montré que la digue était dans un état structurel variable. Les niveaux de crête de digues pensés en 1929 n'étaient pas non plus en rapport avec la connaissance des phénomènes extrêmes. Une partie de la digue sud de la Faute-sur-Mer est à la cote $4 \mathrm{~m}$ NGF $(800 \mathrm{~m})$. 


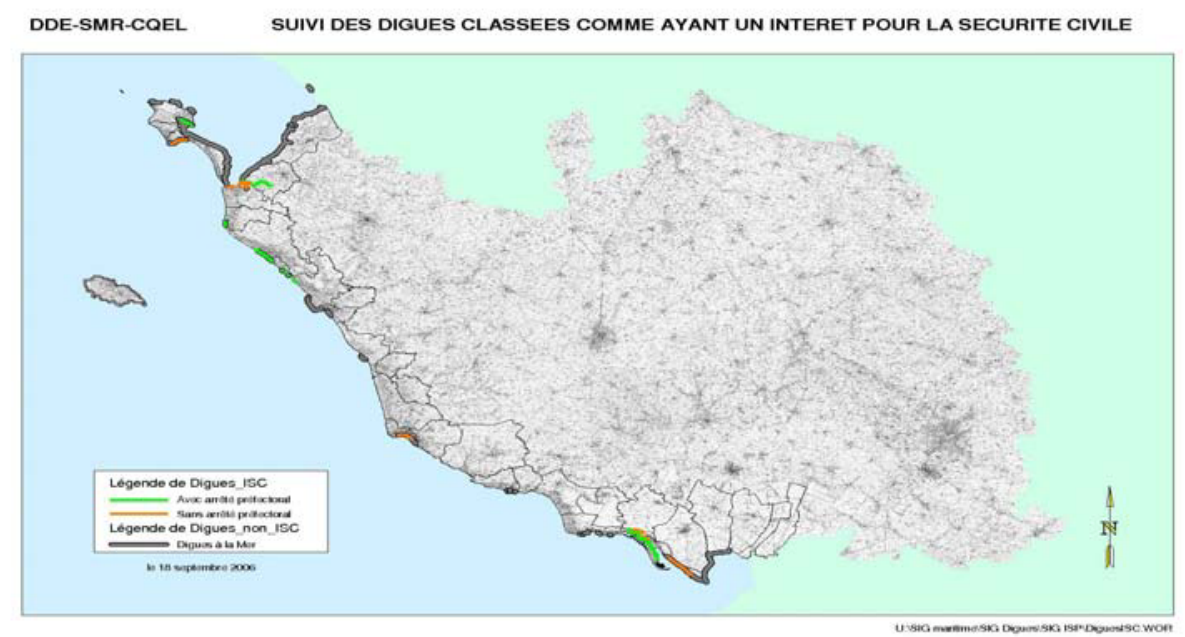

Figure 7. Suivi des digues classées comme ayant un intérêt pour la sécurité civile (DDE de la Vendée, 2006)

Lors de la tempête du 10 mars 2008, associée à une marée de coefficient 106, les niveaux d'eau dans l'estuaire ont atteint 3.7 m NGF. Le système de surveillance mis en place par la commune a permis l'information des populations. Un lourd programme de réhabilitation est en cours sur cet ouvrage. La démarche de classement initiée à La Faute-sur-Mer a été généralisée à $42 \mathrm{~km}$ le long du littoral. A partir de 2005 plus de 16 digues ont fait l'objet de diagnostics. La quasi-totalité des digues classée en 2005 est aujourd'hui couverte par un arrêté préfectoral de classement. La liste de ces digues est examinée régulièrement par l'autorité préfectorale pour un suivi des études et des travaux (voir figure 7).

\subsection{Le décret du 11 décembre 2007, une continuation des efforts de l'Etat pour la sécurité des populations}

La démarche enclenchée par la DDE de la Vendée dès 2005 se poursuit actuellement par celle détaillée fin 2007 dans le décret $n^{\circ}$ 2007-1735 relatif à la sécurité des ouvrages hydrauliques et concernant le classement des digues et barrages. Les méthodes de définition des hauteurs de submersion et des populations exposées sont celles retenues en Vendée. Un nouveau plan de travail démarre pour toutes les digues du département de la Vendée.

\section{Conclusion :}

Les nouvelles règles de classement imposées par le décret du 11 décembre 2007 sur le classement des digues et des barrages reprend en grande partie les éléments de classement initiés en Vendée tout en étant toujours plus spécialisé dans les digues fluviales que dans les digues maritimes. Le classement mis en place en 2005 perdurera en parallèle du nouveau classement qui lui renforce le suivi des digues de retraite. 